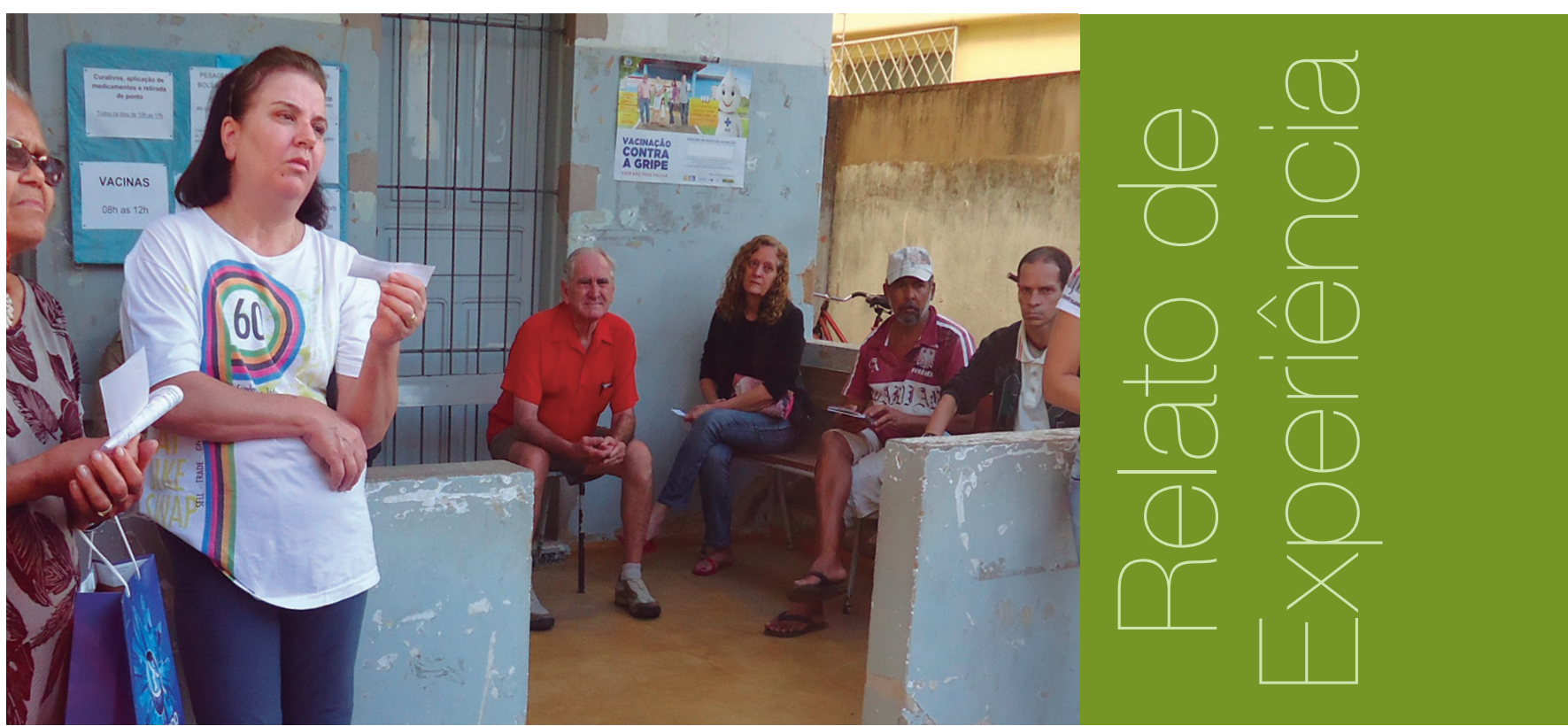

\title{
Educação em saúde: a abordagem sobre doenças sexualmente transmissiveis em salas de espera
}

Katy Andrade Monteiro Zacaron¹ - katy.andrade@ufjf.edu.br

Cristiane Diniz ${ }^{2}$ - colufmg@yahoo.com.br

Juliana Santana Lazarini ${ }^{3}$ - julianas.94@hotmail.com

Luiz Eduardo de Almeida4 - luiz.almeida@ufjf.edu.br

\section{RESUMO}

0 presente relato de experiência buscou evidenciar a experiência do "Projeto de Extensão Sala de Espera: enfoque multiprofissional" na abordagem da temática DST/Sífilis em salas de espera de Unidades de Atenção Primária a Saúde. Dinamizado sob perspectiva crítica e reflexiva, desprende-se desta experiência o êxito da extensão universitária no fornecimento de ações promotoras de saúde e como instrumento indutório na contextualização de discentes de seus reais papeis como futuros profissionais de saúde.

\section{PALAVRAS-CHAVE}

Educação em Saúde. Extensão universitária. Sala de espera. Doenças sexualmente transmissíveis. Sífilis.

\footnotetext{
1 Mestre em Ciência da Reabilitação - Professora do Departamento de Fisioterapia da Universidade Federal de Juiz de Fora Campus Avançado de Governador Valadares, MG.

2 Acadêmica do curso de Medicina da Universidade Federal de Juiz de Fora - Campus Avançado de Governador Valadares, MG.

3 Acadêmica do curso de Nutrição da Universidade Federal de Juiz de Fora - Campus Avançado de Governador Valadares, MG.

4 Mestre em Clínica Odontológica - Professor do Departamento de Odontologia da Universidade Federal de Juiz de Fora - Campus Avançado de Governador Valadares, MG
} 


\section{ABSTRACT}

This experience report aimed to highlight the experience of the "Extension Project Waiting Room: multidisciplinary approach" in the thematic approach STD/Syphilis in waits rooms/ UAPS. Streamlined under critical and reflective perspective, detaches itself from this experience the success of the university extension in the provision of health-promoting actions and how indutório instrument in the context of students of their actual roles as future health professionals.

\section{KEYWORDS}

Health Education . Academic extension. Waiting room. Sexually Transmitted Diseases. Syphilis.

\section{Relato de experiência}

Conforme as novas Diretrizes Curriculares Nacionais, os cursos de graduação da área da saúde devem ofertar ações promotoras da integração ensino-serviço-comunidade (FERREIRA, FORSTER; SANTOS, 2012). Sob esta concepção, galgando um aprendizado que ultrapasse as barreiras físicas da universidade e se concretize no âmbito da comunidade, foi idealizado, em 2014, o "Projeto de Extensão Sala de Espera: enfoque multiprofissional/PESE", sendo delineado em uma perspectiva multiprofissional, envolvendo docentes e discentes dos cursos de Farmácia, Fisioterapia, Medicina, Nutrição e Odontologia da Universidade Federal de Juiz de Fora/Campus Avançado de Governador Valadares (UFJF/GV).

Quanto ao local de atuação, o PESE se fez presente em "Salas de Espera/SE" de Unidades de Atenção Primária à Saúde/UAPS (Bairros Centro, Lourdes e Vila Mariana) de Governador Valadares, MG. A eleição deste cenário se justifica pelo fato de a sala de espera configurar-se, segundo Santos et al. (2012), em um lugar pouco utilizado pelas instituições de saúde, mesmo diante do frequente tempo ocioso de espera das consultas pelos pacientes que ali se fazem presentes. Os autores ainda destacam que estes espaços podem prestar-se a importantes momentos de trocas entre saberes científicos e populares, por meio da implantação de programas de promoção de saúde.

De acordo com Garcia et al. (2007), a SE se consubstancia em um espaço privilegiado da multiprofissionalidade, que exige a atuação em equipe, a responsabilização e 0 vínculo com os usuários. Indo além, pode-se afirmar que dinamizar espaços do serviço com a extensão universitária é levar o conhecimento científico de forma prática e acessível à população, ao mesmo tempo em que fornece aos alunos uma experiência precoce na comunidade (ALMEIDA, 2009).

Permeado ao seu objetivo, o de descrever um relato de experiência da atividade desenvolvida pelo PESE-UFJF/GV, cujo enfoque temático, norteado pelos gestores das UAPS assistidas, foi "Doenças sexualmente transmissíveis - DST's", o presente estudo traz em seu bojo a análise de conteúdo de um fato, sendo organizado sob recorte analítico-transversal e dinamizado sob estratégia narrativa (BELL, 2008). Desprende-se deste percurso a sistematização de desenvolvimento das ações do PESE, extraindo daí três pontos estruturais: o planejamento, a execução e a autoavaliação.

0 sucesso de ações de educação em saúde não se restringe a grandes ideias, na verdade, 0 fundamental é desenvolver meios para alcançá-lo, ou seja, perceber a importância de se planejar (ALMEIDA, 2009). Durante o planejamento, o primeiro ponto trabalhado pela equipe do PESE foi a definição do tema a ser abordado. Direcionados pela gerência administrativa do serviço, buscando assim atender as reais necessidades das comunidades adstritas às UAPS, destacou-se a importância de se discutir a temática "Doenças Sexualmente Transmissíveis/ DST", sendo ainda enfocada a alta prevalência de casos de sífilis.

Confrontando esta realidade com a literatura, constata-se que as DST requerem, além do tratamento individualizado, trabalhos de prevenção e ações de promoção de saúde para que se consiga uma efetiva redução da incidência dessas doenças (Rodrigues et al.,2011). Aprofundando, como exposto por Araújo et al. (2012) e Magalhães et al. (2013), a sífilis destaca-se dentre as DST por acarretar problemas sociais, econômicos e sanitários. No Brasil, em 2011, registrou-se uma taxa de cinco casos de sífilis por mil nascidos vivos no Brasil (BRASIL, 2012). 
Posteriormente, com o intuito de se estabelecer um contato direto com os usuários, bem como direcionar a equipe do PESE para a construção da dinâmica interativa, urnas foram instaladas nas UAPS para que os usuários depositassem suas principais inquietações frente ao assunto a ser abordado, destacando como as mais frequentes: "O que é DST?; Usar camisinha previne contra todas as DST?; Como é feito o tratamento para DST?; É muito complicado?; É possivel pegar DST pelo beijo?; Quem pode pegar?; Quais são os tipos de DST?; Tem cura?; Se eu estiver grávida, eu posso passar para meu filho?". Ressalta-se deste percurso a saída do comum e antidialógico desenvolvimento da extensão, ensino-centrado, para o encontro de um cenário extensionista de "via de mão dupla", que visa em sua existência a troca de experiências entre ensino e serviço em prol da comunidade (ALMEIDA, 2009; FREIRE, 2006).

Concluído o direcionado plano de ação, iniciou-se a execução das atividades. Com média de duração de 30 minutos, foram abordadas as questões supracitadas, bem como outras interpelações surgidas durante a atividade. Desta dinâmica foi construído e afixado um painel interativo - designado, pela equipe, como "Cantinho interativo/UFJF/UAPS" (Figuras 1, 2 e 3). Afirma-se que este modelo pedagógico vai de encontro ao explanado por Cervera, Parrera e Goulart (2011), que destacam 0 importante papel da educação em saúde na capacitação da comunidade como agentes promotores de saúde, ou seja, "empoderando" os indivíduos a serem capazes de se autoperceberem e, principalmente, de cuidarem da sua própria saúde.

Por fim, porém não menos importante, descreve-se a autoavaliação. De forma crítica e reflexiva foram expostos pela equipe os pontos positivos e negativos por eles percebidos durante todo 0 processo. 0 quantitativo de usuários, a receptividade e/ou participação da equipe profissional da UAPS, a dinâmica e a presença do corpo docente foram os principais pontos positivos apontados pelos discentes. Quanto aos pontos negativos, as dificuldades centraram-se no tema, na pouca participação dos usuários e nos ruídos do ambiente. Por fim, de posse destas reflexões, ponderando os pontos positivos e negativos, a equipe do PESE-UFJF/GV caracterizou a atividade como exitosa, entretanto, foi consenso dos membros que o sucesso da ação não esteve apenas atrelado aos acertos, pelo contrário, a discussão sobre os erros foi fundamental para um aprendizado mais coerente com a realidade.

Em síntese, pode-se afirmar que o espaço sala de espera se consagra como um território dinâmico para atividades extensionistas, onde diferentes indivíduos aguardam seu atendimento de saúde, constituindo-se, portanto, em um espaço fértil para implantação de ações educativas, que podem contribuir significativamente para a promoção da saúde, prevenção de agravos e encaminhamento para outras atividades, portanto, encorpando e otimizando ainda mais o papel da atenção primária junto aos serviços de saúde prestados pelo Sistema Único de Saúde, SUS.

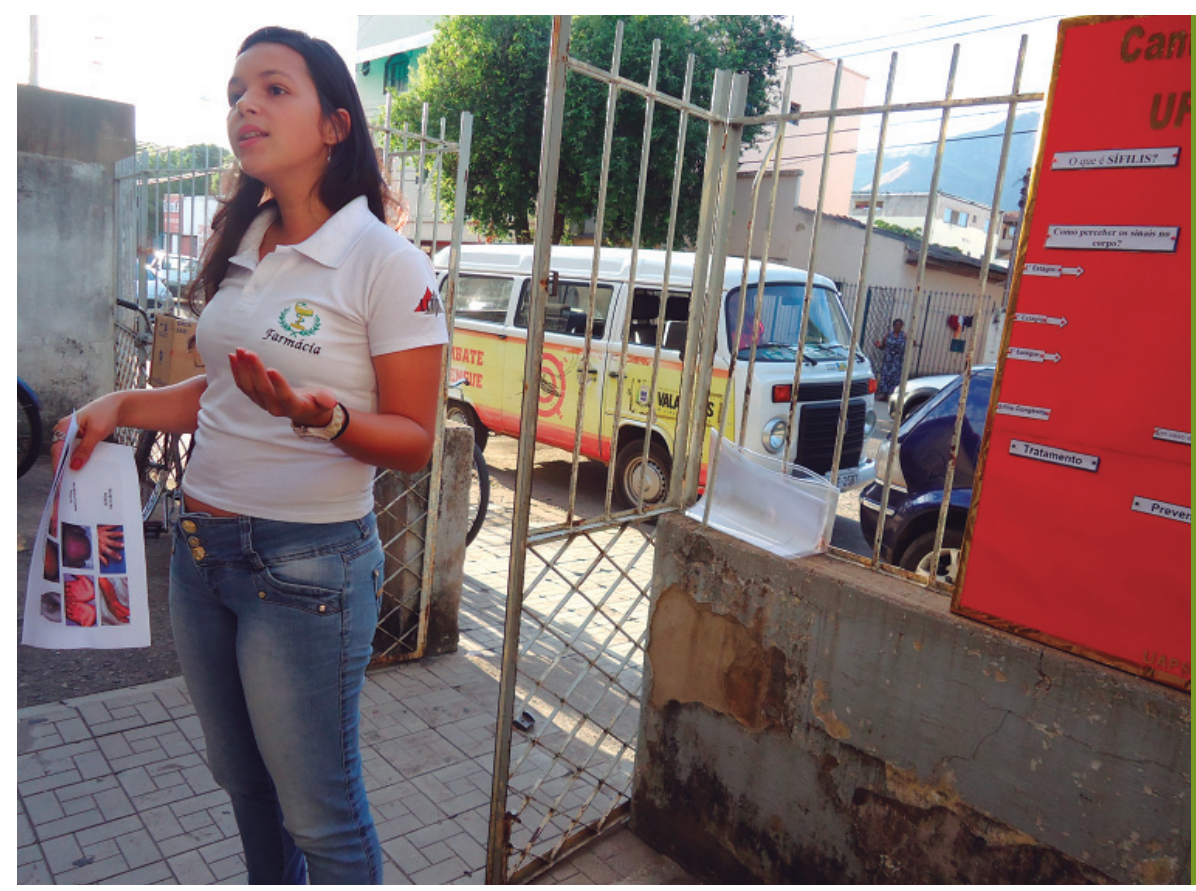

Figura 1: Dinâmica interativa abordando o tema "DST/Sífilis".

Fonte: Projeto de Extensão Sala de Espera: enfoque multiprofissional, 2014 
interativa abordando o tema "DST/Sífilis".

Fonte: Projeto de Extensão Sala de Espera: enfoque multiprofissional, 2014

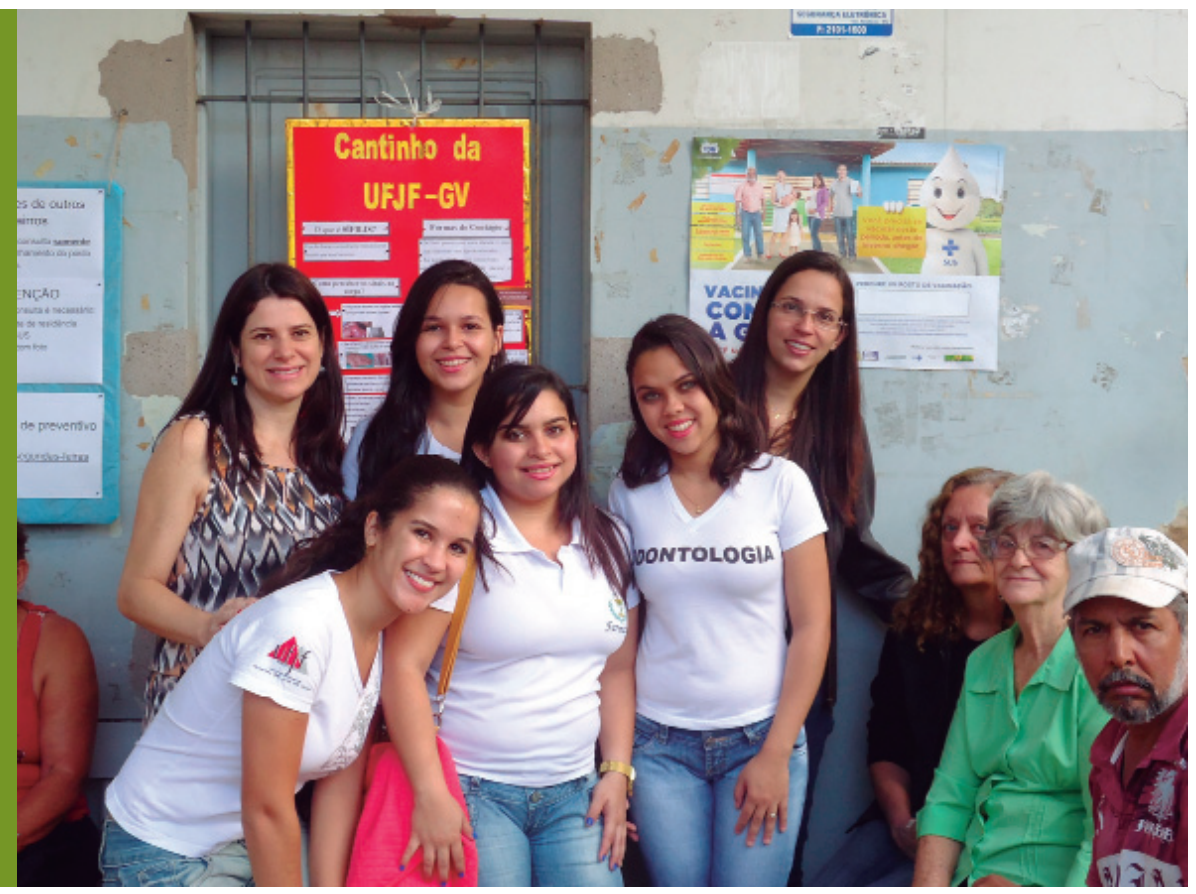

Figura 3: Dinâmica interativa abordando o tema "DST/Sífilis".

Fonte: Projeto de Extensão Sala de Espera: enfoque multiprofissional, 2014

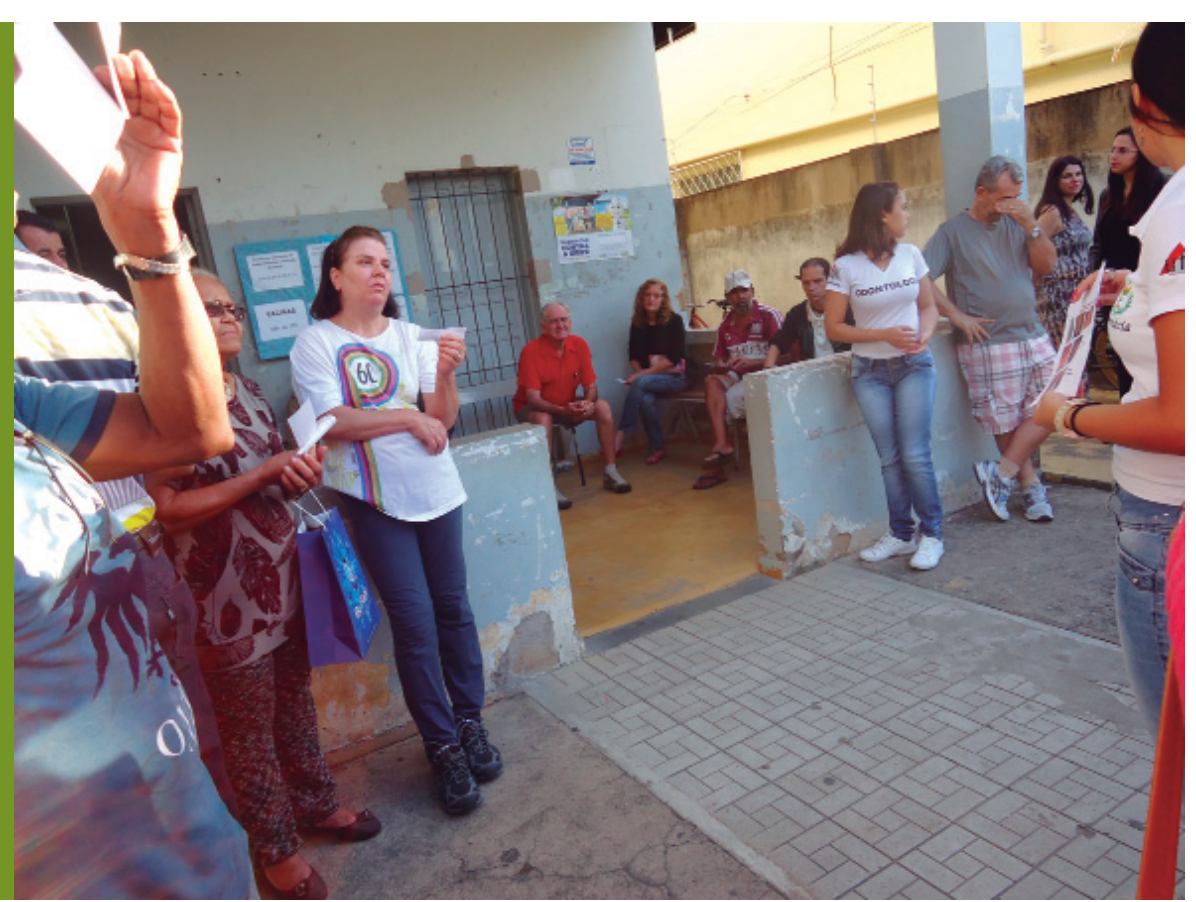

\section{Referências}

ALMEIDA, L.E. Pró-Saúde: Ensino, Pesquisa e Extensão. 1. ed. Juiz de Fora: Editar, 2009.

ARAÚJO, C.L. et al. Incidência da sífilis congênita no Brasil e sua relação com a Estratégia Saúde da Família. Rev Saúde Pública, 46(3):479-486, 2012.

BELL, J. Projeto de Pesquisa: guia para pesquisadores iniciantes em educação, saúde e ciências sociais. 4. ed. Porto Alegre: Artmed, 2008.

BRASIL. Ministério da Saúde. Secretaria de Vigilância em Saúde. Departamento de DST, Aids e Hepatites Virais. Boletim Epidemiológico - Sífilis 2012. Brasília-DF, 2012.

CERVERA, D.P.P.; PARREIRA, B.D.M.; GOULART, B.F. Educação em saúde: percepção dos enfermeiros da atenção básica em Uberaba (MG). Ciência \& Saúde Coletiva, 16(Supl.I): 1547-1554, 2011. 
FERREIRA, J.B.B.; FORSTER, A.C.; SANTOS, J.S. Reconfigurando a interação entre ensino, serviço e comunidade. Rev. bras. educ. med., 36(1): 127-133, 2012.

FREIRE, P. Extensão ou Comunicação. 13. ed. São Paulo: Paz e Terra. 2006.

GARCIA, M.A.A. et al. A Interdisciplinaridade Necessária à Educação Médica. Rev. bras. educ. med., 31 (2): 147-155, 2007.

MAGALHÃES, D.M.S.; KAWAGUCHI, I.A.L.; DIAS, A.; CALDERON, I.M.P. Sífilis materna e congênita: ainda um desafio. Cad. Saúde Pública, 29(6): 1109-1120, 2013.

RODRIGUES, L.M.C. et al. Abordagem às Doenças Sexualmente Transmissíveis em Unidades Básicas de Saúde da Família. Cogitare Enferm., 16(1):63-69, 2011.

SANTOS, D.S. et al. Sala de espera para gestantes: uma estratégia de educação em saúde. Rev. bras. educ. med., 36(1): 62-67, 2012. 